\title{
CONVERGENCE OF MULTIPLE FOURIER SERIES OF FUNCTIONS OF BOUNDED GENERALIZED VARIATION
}

\author{
USHANGI GOGINAVA AND ARTUR SAHAKIAN
}

\begin{abstract}
The paper introduces a new concept of $\Lambda$-variation of multivariable functions and investigates its connection with the convergence of multidimensional Fourier series
\end{abstract}

\section{Classes of Functions of Bounded Generalized Variation}

In 1881 Jordan 11 introduced a class of functions of bounded variation and applied it to the theory of Fourier series. Hereafter this notion was generalized by many authors (quadratic variation, $\Phi$-variation, $\Lambda$-variation ets., see [2, 17, 15, 12]). In two dimensional case the class BV of functions of bounded variation was introduced by Hardy [10].

For an interval $T=[a, b] \subset R$ we denote by $T^{d}=[a, b]^{d}$ the d-dimensional cube in $R^{d}$.

Consider a function $f(x)$ defined on $T^{d}$ and a collection of intervals

$$
J^{k}=\left(a^{k}, b^{k}\right) \subset T, \quad k=1,2, \ldots d .
$$

For $d=1$ we set

$$
f\left(J^{1}\right):=f\left(b^{1}\right)-f\left(a^{1}\right) .
$$

If for any function of $d-1$ variables the expression $f\left(J^{1} \times \cdots \times J^{d-1}\right)$ is already defined, then for a function of $d$ variables the mixed difference is defined as follows:

$$
f\left(J^{1} \times \cdots \times J^{d}\right):=f\left(J^{1} \times \cdots \times J^{d-1}, b^{d}\right)-f\left(J^{1} \times \cdots \times J^{d-1}, a^{d}\right) .
$$

Let $E=\left\{I_{k}\right\}$ be a collection of nonoverlapping intervals from $T$ ordered in arbitrary way and let $\Omega=\Omega(T)$ be the set of all such collections $E$. We denote by $\Omega_{n}=\Omega_{n}(T)$ set of all collections of $n$ nonoverlapping intervals $I_{k} \subset T$.

For sequences of positive numbers

$$
\Lambda^{j}=\left\{\lambda_{n}^{j}\right\}_{n=1}^{\infty}, \quad \lim _{n \rightarrow \infty} \lambda_{n}^{j}=\infty, \quad j=1,2, \ldots, d,
$$

${ }^{0} 2010$ Mathematics Subject Classification: 42B05

Key words and phrases: Multiple Fourier series, Bounded $\Lambda$-variation, Uniform convergence . 
and for a function $f(x), x=\left(x_{1}, \ldots, x_{d}\right) \in T^{d}$ the $\left(\Lambda^{1}, \ldots, \Lambda^{d}\right)$-variation of $f$ with respect to the index set $D:=\{1,2, \ldots, d\}$ is defined as follows:

$$
\left\{\Lambda^{1}, \ldots, \Lambda^{d}\right\} V^{D}\left(f, T^{d}\right):=\sup _{\left\{I_{i_{j}}^{j}\right\} \in \Omega} \sum_{i_{1}, \ldots, i_{d}} \frac{\left|f\left(I_{i_{1}}^{1} \times \cdots \times I_{i_{d}}^{d}\right)\right|}{\lambda_{i_{1}}^{1} \cdots \lambda_{i_{d}}^{d}} .
$$

For an index set $\alpha=\left\{j_{1}, \ldots, j_{p}\right\} \subset D$ and any $x=\left(x_{1}, \ldots, x_{d}\right) \in R^{d}$ we set $\widetilde{\alpha}:=D \backslash \alpha$ and denote by $x_{\alpha}$ the vector of $R^{p}$ consisting of components $x_{j}, j \in \alpha$, i.e.

$$
x_{\alpha}=\left(x_{j_{1}}, \ldots, x_{j_{p}}\right) \in R^{p} .
$$

By

$$
\left\{\Lambda^{j_{1}}, \ldots, \Lambda^{j_{p}}\right\} V^{\alpha}\left(f, x_{\widetilde{\alpha}}, T^{d}\right) \quad \text { and } \quad f\left(I_{i_{j_{1}}}^{1} \times \cdots \times I_{i_{j_{p}}}^{p}, x_{\widetilde{\alpha}}\right)
$$

we denote respectively the $\left(\Lambda^{j_{1}}, \ldots, \Lambda^{j_{p}}\right)$-variation over the $p$-dimensional cube $T^{p}$ and mixed difference of $f$ as a function of variables $x_{j_{1}}, \ldots, x_{j_{p}}$ with fixed values $x_{\tilde{\alpha}}$ of other variables. The $\left(\Lambda^{j_{1}}, \ldots, \Lambda^{j_{p}}\right)$-variation of $f$ with respect to the index set $\alpha$ is defined as follows:

$$
\left\{\Lambda^{j_{1}}, \ldots, \Lambda^{j_{p}}\right\} V^{\alpha}\left(f, T^{p}\right)=\sup _{x_{\widetilde{\alpha}} \in T^{d-p}}\left\{\Lambda^{j_{1}}, \ldots, \Lambda^{j_{p}}\right\} V^{\alpha}\left(f, x_{\widetilde{\alpha}}, T^{d}\right) .
$$

Definition 1. We say that the function $f$ has total Bounded $\left(\Lambda^{1}, \ldots, \Lambda^{d}\right)$ variation on $T^{d}$ and write $f \in\left\{\Lambda^{1}, \ldots, \Lambda^{d}\right\} B V\left(T^{d}\right)$, if

$$
\left\{\Lambda^{1}, \ldots, \Lambda^{d}\right\} V\left(f, T^{d}\right):=\sum_{\alpha \subset D}\left\{\Lambda^{1}, \ldots, \Lambda^{d}\right\} V^{\alpha}\left(f, T^{d}\right)<\infty .
$$

Definition 2. We say that the function $f$ is continuous in $\left(\Lambda^{1}, \ldots, \Lambda^{d}\right)$ variation on $T^{d}$ and write $f \in C\left\{\Lambda^{1}, \ldots, \Lambda^{d}\right\} V\left(T^{d}\right)$, if

$$
\lim _{n \rightarrow \infty}\left\{\Lambda^{j_{1}}, \ldots, \Lambda^{j_{k-1}}, \Lambda_{n}^{j_{k}}, \Lambda^{j_{k+1}}, \ldots, \Lambda^{j_{p}}\right\} V^{\alpha}\left(f, T^{d}\right)=0, \quad k=1,2, \ldots, p
$$

for any $\alpha \subset D, \alpha:=\left\{j_{1}, \ldots, j_{p}\right\}$, where $\Lambda_{n}^{j_{k}}:=\left\{\lambda_{s}^{j_{k}}\right\}_{s=n}^{\infty}$.

Definition 3. We say that the function $f$ has Bounded Partial $\left(\Lambda^{1}, \ldots, \Lambda^{d}\right)$ variation and write $f \in P\left\{\Lambda^{1}, \ldots, \Lambda^{d}\right\} B V\left(T^{d}\right)$ if

$$
P\left\{\Lambda^{1}, \ldots, \Lambda^{d}\right\} V\left(f, T^{d}\right):=\sum_{i=1}^{d} \Lambda^{i} V^{\{i\}}\left(f, T^{d}\right)<\infty .
$$

In the case $\Lambda^{1}=\cdots=\Lambda^{d}=\Lambda$ we set

$$
\begin{array}{rlrl}
\Lambda B V\left(T^{d}\right):= & & \left\{\Lambda^{1}, \ldots, \Lambda^{d}\right\} B V\left(T^{d}\right), \\
C \Lambda V\left(T^{d}\right):= & & C\left\{\Lambda^{1}, \ldots, \Lambda^{d}\right\} V\left(T^{d}\right), \\
P \Lambda B V\left(T^{d}\right) & := & & P\left\{\Lambda^{1}, \ldots, \Lambda^{d}\right\} B V\left(T^{d}\right) .
\end{array}
$$


If $\lambda_{n} \equiv 1$ (or if $0<c<\lambda_{n}<C<\infty, n=1,2, \ldots$ ) the classes $\Lambda B V$ and $P \Lambda B V$ coincide with the Hardy class $B V$ and $P B V$ respectively. Hence it is reasonable to assume that $\lambda_{n} \rightarrow \infty$.

When $\lambda_{n}=n$ for all $n=1,2 \ldots$ we say Harmonic Variation instead of $\Lambda$-variation and write $H$ instead of $\Lambda$, i.e. $H B V, P H B V, C H V$, ets.

For two variable functions Dyachenko and Waterman [5] introduced another class of functions of generalized bounded variation.

Denoting by $\Gamma$ the the set of finite collections of nonoverlapping rectangles $A_{k}:=\left[\alpha_{k}, \beta_{k}\right] \times\left[\gamma_{k}, \delta_{k}\right] \subset T^{2}$, for a function $f(x, y), x, y \in T$, we set

$$
\Lambda^{*} V\left(f, T^{2}\right):=\sup _{\left\{A_{k}\right\} \in \Gamma} \sum_{k} \frac{\left|f\left(A_{k}\right)\right|}{\lambda_{k}} .
$$

Definition 4 (Dyachenko, Waterman). We say that $f \in \Lambda^{*} B V\left(T^{2}\right)$ if

$$
\Lambda V\left(f, T^{2}\right):=\Lambda V_{1}\left(f, T^{2}\right)+\Lambda V_{2}\left(f, T^{2}\right)+\Lambda^{*} V\left(f, T^{2}\right)<\infty .
$$

In this paper we introduce a new classes of functions of generalized bounded variation and investigate the convergence of Fourier series of function of that classes.

For the sequence $\Lambda=\left\{\lambda_{n}\right\}_{n=1}^{\infty}$ we denote

$$
\Lambda^{\#} V_{s}\left(f, T^{d}\right):=\sup _{\left\{x^{i}\{s\}\right\} \subset T^{d-1}} \sup _{\left\{I_{i}^{s}\right\} \in \Omega} \sum_{i} \frac{\left|f\left(I_{i}^{s}, x^{i}\{s\}\right)\right|}{\lambda_{i}},
$$

where

$$
x^{i}\{s\}:=\left(x_{1}^{i}, \ldots, x_{s-1}^{i}, x_{s+1}^{i}, \ldots, x_{d}^{i}\right) \quad \text { for } \quad x^{i}:=\left(x_{1}^{i}, \ldots, x_{d}^{i}\right) .
$$

Definition 5. We say that the function $f$ belongs to the class $\Lambda^{\#} B V\left(T^{d}\right)$, if

$$
\Lambda^{\#} V\left(f, T^{d}\right):=\sum_{s=1}^{d} \Lambda^{\#} V_{s}\left(f, T^{d}\right)<\infty .
$$

The notion of $\Lambda$-variation was introduced by Waterman [15] in one dimensional case, by Sahakian [14] in two dimensional case and by Sablin [13] in the case of higher dimensions. The notion of bounded partial variation (class $P B V$ ) was introduced by Goginava in [7. These classes of functions of generalized bounded variation play an important role in the theory Fourier series.

Remark 1. It is not hard to see that $\Lambda^{\#} B V\left(T^{d}\right) \subset P \Lambda B V\left(T^{d}\right)$ for any $d>1$ and $\Lambda^{*} B V\left(T^{2}\right) \subset \Lambda^{\#} B V\left(T^{2}\right)$.

We prove that the following theorem is true.

Theorem 1. Let $d \geq 2$ and $T=\left(t_{1}, t_{2}\right) \subset R$. If

$$
\Lambda=\left\{\lambda_{n}\right\} \quad \text { with } \quad \lambda_{n}=\frac{n}{\log ^{d-1}(n+1)}, \quad n=1,2, \ldots,
$$


then

$$
H V\left(f, T^{d}\right) \leq M(d) \Lambda^{\#} V\left(f, T^{d}\right)
$$

Proof. We have to prove that for any $\alpha:=\left\{j_{1}, \ldots, j_{p}\right\} \subset D$

$$
\sup _{\left\{I_{i_{j}}^{j}\right\} \in \Omega} \sum_{i_{1}, \ldots, i_{p}} \frac{\left|f\left(I_{i_{1}}^{1} \times \cdots \times I_{i_{p}}^{p}, x_{\widetilde{\alpha}}\right)\right|}{i_{1} \cdots i_{p}} \leq M(d) \sum_{s=1}^{d} \Lambda^{\#} V_{s}\left(f, T^{d}\right)
$$

To this end, observe that

$$
\begin{aligned}
& \sum_{i_{1}, \ldots, i_{p}} \frac{\left|f\left(I_{i_{1}}^{1} \times \cdots \times I_{i_{p}}^{p}, x_{\widetilde{\alpha}}\right)\right|}{i_{1} \cdots i_{p}} \\
= & \sum_{\sigma} \sum_{i_{\sigma(1)} \leq \cdots \leq i_{\sigma(p)}} \frac{\left|f\left(I_{i_{1}}^{1} \times \cdots \times I_{i_{p}}^{p}, x_{\widetilde{\alpha}}\right)\right|}{i_{1} \cdots i_{p}}
\end{aligned}
$$

where the sum is taken over all rearrangements $\sigma=\{\sigma(k)\}_{k=1}^{p}$ of the set $\{1,2, \ldots, p\}$.

Next, we have

$$
\begin{gathered}
\sum_{i_{1} \leq \cdots \leq i_{p}} \frac{\left|f\left(I_{i_{1}}^{1} \times \cdots \times I_{i_{p}}^{p}, x_{\widetilde{\alpha}}\right)\right|}{i_{1} \cdots i_{p}} \\
=\sum_{i_{p}} \frac{1}{i_{p}} \sum_{i_{1} \leq \cdots \leq i_{p}} \frac{\left|f\left(I_{i_{1}}^{1} \times \cdots \times I_{i_{p}}^{p}, x_{\widetilde{\alpha}}\right)\right|}{i_{1} \cdots i_{p-1}} .
\end{gathered}
$$

Taking into account that for the fixed $i_{p}\left(i_{1} \leq \ldots \leq i_{p}\right)$ there exists $x_{1}^{i_{p}}, \ldots, x_{p-1}^{i_{p}} \in$ $T$ such that

$$
\left|f\left(I_{i_{1}}^{1} \times \cdots \times I_{i_{p}}^{p}, x_{\widetilde{\alpha}}\right)\right| \leq 2^{d}\left|f\left(I_{i_{p}}^{p}, x_{1}^{i_{p}}, \ldots, x_{p-1}^{i_{p}}, x_{\widetilde{\alpha}}\right)\right|
$$

from (66) we obtain

$$
\begin{aligned}
& \sum_{i_{1} \leq \cdots \leq i_{p}} \frac{\left|f\left(I_{i_{1}}^{1} \times \cdots \times I_{i_{p}}^{p}, x_{\widetilde{\alpha}}\right)\right|}{i_{1} \cdots i_{p}} \\
\leq & 2^{d} \sum_{i_{p}} \frac{\left|f\left(I_{i_{p}}^{p}, x_{1}^{i_{p}}, \ldots, x_{p-1}^{i_{p}}, x_{\widetilde{\alpha}}\right)\right|}{i_{p}} \sum_{i_{1} \leq \cdots \leq i_{p}} \frac{1}{i_{1} \cdots i_{p-1}} \\
\leq & M(d) \sum_{i_{p}} \frac{\log ^{d-1}\left(i_{p}+1\right)}{i_{p}}\left|f\left(I_{i_{p}}^{p}, x_{1}^{i_{p}}, \ldots, x_{p-1}^{i_{p}}, x_{\widetilde{\alpha}}\right)\right| \\
\leq & M(d) \Lambda^{\#} V_{i_{p}}\left(f, T^{d}\right) \leq M(d) \Lambda^{\#} V\left(f, T^{d}\right) .
\end{aligned}
$$


Similarly one can obtain bounds for other summands in the right hind side of (5), which imply (3). Theorem 1 is proved.

Corollary 1. If the sequence $\Lambda$ is defined by (2), then $\Lambda^{\#} B V\left(T^{d}\right) \subset H B V\left(T^{d}\right)$.

Now, we denote

$$
\Delta:=\left\{\delta=\left(\delta_{1}, \ldots, \delta_{d}\right): \delta_{i}= \pm 1, i=1,2, \ldots, d\right\}
$$

and

$$
\pi_{\varepsilon \delta}(x):=\left(x_{1}, x_{1}+\varepsilon \delta_{1}\right) \times \cdots \times\left(x_{d}, x_{d}+\varepsilon \delta_{d}\right),
$$

for $x=\left(x_{1}, \ldots, x_{d}\right) \in R^{d}$ and $\varepsilon>0$. We set $\pi_{\delta}(x):=\pi_{\varepsilon \delta}(x)$, if $\varepsilon=1$.

For a function $f$ defined in some neighbourhood of a point $x$ and $\delta \in \Delta$ we set

$$
f_{\delta}(x):=\lim _{t \in \pi_{\delta}(x), t \rightarrow x} f(t)
$$

if the last limit exists.

Theorem 2. Suppose $f \in \Lambda^{\#} B V\left(T^{d}\right)$ for some sequence $\Lambda=\left\{\lambda_{n}\right\}$.

a) If the limit $f_{\delta}(x)$ exists for some $x=\left(x_{1}, \ldots, x_{d}\right) \in T^{d}$ and some $\delta=\left(\delta_{1}, \ldots, \delta_{d}\right) \in \Delta$, then

$$
\lim _{\varepsilon \rightarrow 0} \Lambda^{\#} V\left(f, \pi_{\varepsilon \delta}(x)\right)=0 .
$$

b) If $f$ is continuous on some compact $K \subset T^{d}$, then

$$
\lim _{\varepsilon \rightarrow 0} \Lambda^{\#} V\left(f,\left[x_{1}-\varepsilon, x_{1}+\varepsilon\right] \times \cdots \times\left[x_{d}-\varepsilon, x_{d}+\varepsilon\right]\right)=0
$$

uniformly with respect to $x=\left(x_{1}, \ldots, x_{d}\right) \in K$.

Proof. According to Definition 5, we need to prove that

$$
\lim _{\varepsilon \rightarrow 0} \Lambda^{\#} V_{s}\left(f, \pi_{\varepsilon \delta}(x)\right)=0
$$

for any $s=1,2, \ldots, d$. Without loss of generality we can assume that $s=1$ and $\delta_{i}=1$ for $i=1,2, \ldots, d$. Assume to the contrary that (11) does not holds:

$$
\lim _{\varepsilon \rightarrow 0} \Lambda^{\#} V_{1}\left(f, \pi_{\varepsilon \delta}(x)\right) \neq 0 .
$$

Then there exists a number $\alpha$ such that

$$
\Lambda^{\#} V_{1}\left(f, \pi_{\varepsilon \delta}(x)\right)>\alpha>0
$$

for any $\varepsilon>0$.

Using induction on $k=1,2, \ldots$, we construct positive numbers $\varepsilon_{k}$ and the sequences of collections of non-overlapping intervals

$$
I_{i}^{1} \subset\left(x_{1}+\varepsilon_{k+1}, x_{1}+\varepsilon_{k}\right), \quad i=n_{k}+1, \ldots, n_{k+1}
$$

and vectors

$$
\beta^{i}=\left(\beta_{1}^{i}, \ldots, \beta_{d}^{i}\right) \in \pi_{\varepsilon_{k} \delta}(x), \quad i=n_{k}+1, \ldots, n_{k+1}
$$


as follows. By (12), for a fixed number $\varepsilon_{1}>0$ we find a collection of nonoverlapping intervals

$$
I_{i}^{1} \subset\left(x_{1}, x_{1}+\varepsilon_{1}\right), \quad i=1, \ldots, n_{1}
$$

and vectors

$$
\beta^{i}=\left(\beta_{1}^{i}, \ldots, \beta_{d}^{i}\right) \in \pi_{\varepsilon_{1} \delta}(x), \quad i=1, \ldots, n_{1}
$$

such that

$$
\sum_{i=1}^{n_{1}} \frac{\left|f\left(I_{i}^{1} ; \beta_{2}^{i}, \ldots, \beta_{d}^{i}\right)\right|}{\lambda_{i}}>\alpha
$$

Now, suppose the number $\varepsilon_{k}$, intervals (13) and the vectors (14) for some $k=1,2 \ldots$ are constructed . Since the limit $f_{\delta}(x)$ exists, we can choose $\varepsilon_{k+1}$ satisfying

$$
0<\varepsilon_{k+1}<\varepsilon_{k}, \quad\left(x_{1}, x_{1}+\varepsilon_{k+1}\right) \bigcap\left(\bigcup_{i=1}^{n_{k}} I_{i}^{1}\right)=\emptyset
$$

and

$$
\sum_{i=1}^{n_{k}} \frac{\left|f\left(J_{i}^{1} ; \gamma_{2}^{i}, \ldots, \gamma_{d}^{i}\right)\right|}{\lambda_{i}}<\frac{\alpha}{2}
$$

for any collection of non-overlapping intervals

$$
J_{i}^{1} \subset\left(x_{1}, x_{1}+\varepsilon_{k+1}\right), \quad i=1, \ldots, n_{k}
$$

and for any vectors

$$
\gamma^{i}=\left(\gamma_{1}^{i}, \ldots, \gamma_{d}^{i}\right) \in \pi_{\varepsilon_{k+1} \delta}(x), \quad i=1, \ldots, n_{k} .
$$

Further, according to (12) there is a collection of non-overlapping intervals

$$
J_{i}^{1} \subset\left(x_{1}, x_{1}+\varepsilon_{k+1}\right), \quad i=1, \ldots, n_{k+1}
$$

and vectors

$$
\gamma^{i}=\left(\gamma_{1}^{i}, \ldots, \gamma_{d}^{i}\right) \in \pi_{\varepsilon_{k+1} \delta}(x), \quad i=1, \ldots, n_{k+1}
$$

such that

$$
\sum_{i=1}^{n_{k+1}} \frac{\left|f\left(J_{i}^{1} ; \gamma_{2}^{i}, \ldots, \gamma_{d}^{i}\right)\right|}{\lambda_{i}}>\alpha
$$

Now, denoting

$$
I_{i}^{1}=J_{i}^{1}, \beta^{i}=\gamma^{i} \quad \text { for } \quad i=n_{k}+1, \ldots, n_{k+1},
$$

from (17) and (19) we get

$$
\sum_{i=n_{k}+1}^{n_{k+1}} \frac{\left|f\left(I_{i}^{1} ; \beta_{2}^{i}, \ldots, \beta_{d}^{i}\right)\right|}{\lambda_{i}}>\frac{\alpha}{2} .
$$

Intervals (13) and vectors (14) for $k=1,2, \ldots$, are constructed. 
By (16), (18) and (20), the intervals $I_{i}^{1}$ are non-overlapping for $i=1,2, \ldots$, while according to (21),

$$
\sum_{i=1}^{\infty} \frac{\left|f\left(I_{i}^{1} ; \beta_{2}^{i}, \ldots, \beta_{d}^{i}\right)\right|}{\lambda_{i}}=\infty
$$

Consequently, $\Lambda^{\#} V_{1}\left(f, T^{d}\right)=\infty$. This contradiction completes proof of the statement a) of Theorem 2 ,

To prove statement b), observe that a) obviously implies (10) for any point $x \in T^{d}$, where $f$ is continuous. Hence, we have to prove that (10) holds uniformly with respect to $x \in K$, provided that $f$ is continuous on the compact $K \subset T^{d}$.

To this end let us assume to the contrary that (10) does not hold uniformly on $K$. Then there exist $\delta>0$ and sequences

$$
x^{i}=\left(x_{1}^{i}, \ldots x_{d}^{i}\right) \in K \text { and } \varepsilon_{i}>0, \quad i=1,2, \ldots \quad \text { with } \quad \varepsilon_{i} \rightarrow 0
$$

such that

$$
\Lambda^{\#} V\left(f ;\left[x_{1}^{i}-\varepsilon_{i}, x_{1}^{i}+\varepsilon_{i}\right] \times \cdots \times\left[x_{d}^{i}-\varepsilon_{i}, x_{d}^{i}+\varepsilon_{i}\right]\right) \geq \delta>0 .
$$

Since $K$ is compact we can assume without loss of generality that $x^{i} \rightarrow x$ for some $x=\left(x_{1}, \ldots, x_{d}\right) \in K$. Then obviously for each $\varepsilon>0$ there is a number $i(\varepsilon)$ such that

$$
\left[x_{j}^{i}-\varepsilon_{i}, x_{j}^{i}+\varepsilon_{i}\right] \subset\left[x_{j}-\varepsilon, x_{j}+\varepsilon\right], \quad j=1, \ldots, d \quad \text { for } \quad i>i(\varepsilon) .
$$

Consequently,

$$
\Lambda^{\#} V\left(f ;\left[x_{1}-\varepsilon, x_{1}+\varepsilon\right] \times \cdots \times\left[x_{d}-\varepsilon, x_{d}+\varepsilon\right]\right) \geq \delta>0,
$$

for any $\varepsilon>0$, which is a contradiction.

Theorem 2 is proved.

Next, we define

$$
v_{s}^{\#}(f, n):=\sup _{\left\{x^{i}\right\}_{i=1}^{n} \subset T^{d}} \sup _{\left\{I_{i}^{s}\right\}_{i=1}^{n} \in \Omega_{n}} \sum_{i=1}^{n}\left|f\left(I_{i}^{s}, x^{i}\{s\}\right)\right|, \quad s=1, \ldots, d, \quad n=1,2, \ldots,
$$

where $x^{i}\{s\}$ is as in (1). The following theorem holds.

Theorem 3. If the function $f(x), x \in T^{d}$ satisfies the condition

$$
\sum_{n=1}^{\infty} \frac{v_{s}^{\#}(f, n) \log ^{d-1}(n+1)}{n^{2}}<\infty, \quad s=1,2, \ldots, d
$$

then $f \in\left\{\frac{n}{\log ^{d-1}(n+1)}\right\}^{\#} B V\left(T^{d}\right)$. 
Proof. Let $s=1, \ldots, d$ be fixed. The for any collection of intervals $\left\{I_{i}^{s}\right\}_{i=1}^{n} \in \Omega_{n}$ and a sequence of vectors $\left\{x^{i}\right\}_{i=1}^{n} \in T^{d}$, using Abel's partial summation we obtain

$$
\begin{aligned}
& \sum_{j=1}^{n} \frac{\left|f\left(I_{j}^{s}, x^{j}\{s\}\right)\right| \log ^{d-1}(j+1)}{j} \\
= & \sum_{j=1}^{n-1}\left(\frac{\log ^{d-1}(j+1)}{j}-\frac{\log ^{d-1}(j+2)}{j+1}\right) \sum_{k=1}^{j}\left|f\left(I_{k}^{s}, x^{k}\{s\}\right)\right| \\
& +\frac{\log ^{d-1}(n+1)}{n} \sum_{j=1}^{n}\left|f\left(I_{j}^{s}, x^{j}\{s\}\right)\right| \\
\leq & \sum_{j=1}^{n-1}\left(\frac{\log ^{d-1}(j+1)}{j}-\frac{\log ^{d-1}(j+2)}{j+1}\right) v_{s}^{\#}(f, j) \\
& +\frac{\log ^{d-1}(n+1)}{n} v_{s}^{\#}(f, n) .
\end{aligned}
$$

Using the inequality

$$
\begin{aligned}
& \frac{\log ^{d-1}(n+1)}{n} v_{s}^{\#}(f, n) \\
\leq & \sum_{j=n}^{\infty}\left(\frac{\log ^{d-1}(j+1)}{j}-\frac{\log ^{d-1}(j+2)}{j+1}\right) v_{s}^{\#}(f, j),
\end{aligned}
$$

from (22) we get

$$
\left\{\frac{n}{\log ^{d-1}(n+1)}\right\}^{\#} V_{s}\left(f, T^{d}\right) \leq c \sum_{n=1}^{\infty} \frac{v_{s}^{\#}(f, n) \log ^{d-1}(n+1)}{n^{2}}<\infty .
$$

Theorem 3 is proved.

\section{Convergence of multiple Fourier series}

We suppose throughout this section, that $T=[0,2 \pi)$ and $T^{d}=[0,2 \pi)^{d}$, $d \geq 2$, stands for the $d$-dimensional torus.

We denote by $C\left(T^{d}\right)$ the space of continuous and $2 \pi$-periodic with respect to each variable functions with the norm

$$
\|f\|_{C}:=\sup _{\left(x_{1}, \ldots, x_{d}\right) \in T^{d}}\left|f\left(x_{1}, \ldots, x_{d}\right)\right| .
$$

The Fourier series of the function $f \in L^{1}\left(T^{d}\right)$ with respect to the trigonometric system is the series

$$
S f\left(x_{1}, \ldots, x_{d}\right):=\sum_{n_{1}, \ldots, n_{d}=-\infty}^{+\infty} \widehat{f}\left(n_{1}, \ldots, n_{d}\right) e^{i\left(n_{1} x_{1}+\cdots+n_{d} x_{d}\right)},
$$


where

$$
\widehat{f}\left(n_{1}, \ldots, n_{d}\right)=\frac{1}{(2 \pi)^{d}} \int_{T^{d}} f\left(x^{1}, \ldots, x^{d}\right) e^{-i\left(n_{1} x_{1}+\cdots+n_{d} x_{d}\right)} d x_{1} \cdots d x_{d}
$$

are the Fourier coefficients of $f$.

In this paper we consider convergence of only rectangular partial sums (convergence in the sense of Pringsheim) of $d$-dimensional Fourier series. Recall that the rectangular partial sums are defined as follows:

$$
\begin{aligned}
& S_{N_{1}, \ldots, N_{d}} f\left(x_{1}, \ldots, x_{d}\right) \\
& :=\sum_{n_{1}=-N_{1}}^{N_{1}} \ldots \sum_{n_{d}=-N_{d}}^{N_{d}} \widehat{f}\left(n_{1}, \ldots, n_{d}\right) e^{i\left(n_{1} x^{1}+\cdots+n_{d} x^{d}\right)} .
\end{aligned}
$$

We say that the point $x \in T^{d}$ is a regular point of a function $f$, if the limit $f_{\delta}(x)$ defined by (8) exists for any $\delta \in \Delta$ (see(7)). For the regular point $x$ we denote

$$
f^{*}(x):=\frac{1}{2^{d}} \sum_{\delta \in \Delta} f_{\delta}(x) .
$$

Definition 6. We say that the class of functions $V \subset L^{1}\left(T^{d}\right)$ is a class of convergence on $T^{d}$, if for any function $f \in V$

1) the Fourier series of $f$ converges to $f^{*}(x)$ at any regular point $x \in T^{d}$,

2) the convergence is uniform on a compact $K \subset T^{d}$, if $f$ is continuous on $K$.

The well known Dirichlet-Jordan theorem (see [18]) states that the Fourier series of a function $f(x), x \in T$ of bounded variation converges at every point $x$ to the value $[f(x+0)+f(x-0)] / 2$. If $f$ is in addition continuous on $T$, then the Fourier series converges uniformly on $T$.

Hardy [10] generalized the Dirichlet-Jordan theorem to the double Fourier series and proved that $B V$ is a class of convergence on $T^{2}$.

The following theorem was proved by Waterman (for $d=1$ ) and Sahakian (for $d=2$ ).

Theorem WS (Waterman [15, Sahakian [14]). If $d=1$ or $d=2$, then the class $H B V\left(T^{d}\right)$ is a class of convergence on $T^{d}$.

In [1] Bakhvalov proved that the class $H B V$ is not a class of convergence on $T^{d}$, if $d>2$. On the other hand, he proved the following theorem.

Theorem B (Bakhvalov [1]). The class $C H V\left(T^{d}\right)$ is a class of convergence on $T^{d}$ for any $d=1,2, \ldots$

Convergence of spherical and other partial sums of double Fourier series of functions of bounded $\Lambda$-variation was investigated in deatails by Dyachenko [3, 4].

In 8, 9] Goginava and Sahakian investigated convergence of multiple Fourier series of functions of bounded partial $\Lambda$-variation. In particular, the following theorem was proved. 
Theorem GS. a) If and $\Lambda=\left\{\lambda_{n}\right\}_{n=1}^{\infty}$ with

$$
\lambda_{n}=\frac{n}{\log ^{d-1+\varepsilon}(n+1)}, \quad n=1,2, \ldots, \quad d>1,
$$

for some $\varepsilon>0$, then the class $P \Lambda B V\left(T^{d}\right)$ is a class of convergence on $T^{d}$. b) If $\Lambda=\left\{\lambda_{n}\right\}_{n=1}^{\infty}$ with

$$
\lambda_{n}=\frac{n}{\log ^{d-1}(n+1)}, \quad n=1,2, \ldots, \quad d>1,
$$

then the class $P \Lambda B V\left(T^{d}\right)$ is not a class of convergence on $T^{d}$.

In [5], Dyachenko and Waterman proved that the class $\Lambda^{*} B V\left(T^{2}\right)$ is a class convergence on $T^{2}$ for $\Lambda=\left\{\lambda_{n}\right\}$ with $\lambda_{n}=\frac{n}{\ln (n+1)}, n=1,2, \ldots$

The main result of the present paper is the following theorem.

Theorem 4. a) If $\Lambda=\left\{\lambda_{n}\right\}_{n=1}^{\infty}$ with

$$
\lambda_{n}=\frac{n}{\log ^{d-1}(n+1)}, \quad n=1,2, \ldots, \quad d>1,
$$

then the class $\Lambda^{\#} B V\left(T^{d}\right)$ is a class of convergence on $T^{d}$.

b)If $\Lambda=\left\{\lambda_{n}\right\}_{n=1}^{\infty}$ with

$$
\lambda_{n}:=\left\{\frac{n \xi_{n}}{\log ^{d-1}(n+1)}\right\}, \quad n=1,2, \ldots \quad d>1,
$$

where $\xi_{n} \rightarrow \infty$ as $n \rightarrow \infty$, then there exists a continuous function $f \in$ $\Lambda^{\#} B V\left(T^{d}\right)$ such that the cubical partial sums of d-dimensional Fourier series of $f$ diverge unboundedly at $(0, \ldots, 0) \in T^{d}$.

Proof of Theorem 4. The proof of the part a) is based on the following statement, that in the case $d=2$ is proved by Sahakian (see formulaes (33) and (35) in [14]). For an arbitrary $d>2$ the proof is similar.

Lemma S. Suppose $f \in H V\left(T^{d}\right)$ and $x \in T^{d}$. If the limit $f_{\delta}(x)$ exists for any $\delta \in \Delta$, then for any $\varepsilon>0$

$$
\left|S_{n_{1}, \ldots, n_{d}} f(x)-f^{*}(x)\right| \leq M(d) \sum_{\delta \in \Delta} H V\left(f ; \pi_{\varepsilon \delta}(x)\right)+o(1),
$$

as $n_{i} \rightarrow \infty, i=1,2, \ldots, d$.

Moreover, the quantity o(1) tends to 0 uniformly on a compact $K$, if $f$ is continuous on $K$.

Now, if the sequence $\Lambda=\left\{\lambda_{n}\right\}$ is defined by (26) and $f \in \Lambda^{\#} B V\left(T^{d}\right)$, then Lemma $\mathrm{S}$ and Theorem 1 imply that for any $\varepsilon>0$

$$
\left|S_{n_{1}, \ldots, n_{d}} f(x)-f^{*}(x)\right| \leq M(d) \sum_{\delta \in \Delta} \Lambda^{\#} V\left(f ; \pi_{\varepsilon \delta}(x)\right)+o(1),
$$

which combined with Theorem 2 completes the proof of a). 
To prove part b) suppose that $\Lambda=\left\{\lambda_{n}\right\}$ is a sequence defined by (27). It is not hard to see that the class $C\left(T^{d}\right) \cap \Lambda^{\#} B V\left(T^{d}\right)$ is a Banach space with the norm

Denoting

$$
\|f\|_{\Lambda \# B V}:=\|f\|_{C}+\Lambda^{\#} B V(f) .
$$

$$
A_{i_{1}, \ldots, i_{d}}:=\left[\frac{\pi i_{1}}{N+1 / 2}, \frac{\pi\left(i_{1}+1\right)}{N+1 / 2}\right) \times \cdots \times\left[\frac{\pi i_{d}}{N+1 / 2}, \frac{\pi\left(i_{d}+1\right)}{N+1 / 2}\right),
$$

we consider the following functions

$$
g_{N}\left(x_{1}, \ldots, x_{d}\right):=\sum_{i_{1}, \ldots, i_{d}=1}^{N-1} 1_{A_{i_{1}, \ldots, i_{d}}}\left(x_{1}, \ldots, x_{d}\right) \prod_{s=1}^{d} \sin (N+1 / 2) x_{s}
$$

for $N=2,3, \ldots$, where $1_{A}\left(x_{1}, \ldots, x_{d}\right)$ is the characteristic function of a set $A \subset T^{d}$.

It is easy to check that

$$
\left\{\frac{n \xi_{n}}{\log ^{d-1}(n+1)}\right\}^{\#} V_{s}\left(g_{N}\right) \leq c \sum_{i=1}^{N-1} \frac{\log ^{d-1}(i+1)}{i \xi_{i}}=o\left(\log ^{d} N\right)
$$

and hence

$$
\left\|g_{N}\right\|_{\Lambda \# B V}=o\left(\log ^{d} N\right)=\eta_{N} \log ^{d} N
$$

where $\eta_{N} \rightarrow 0$ as $N \rightarrow \infty$. Now, setting

$$
f_{N}:=\frac{g_{N}}{\eta_{N} \log ^{d} N}, \quad N=2,3, \ldots,
$$

we obtain that $f_{N} \in \Lambda^{\#} B V\left(T^{d}\right)$ and

$$
\sup _{N}\left\|f_{N}\right\|_{\Lambda \# B V}<\infty \text {. }
$$

Now, for the cubical partial sums of the d-dimensional Fourier series of $f_{N}$ at $(0, \ldots, 0) \in T^{d}$ we have that

$$
\begin{aligned}
& \pi^{d} S_{N, \cdots, N} f_{N}(0, \cdots, 0) \\
= & \frac{1}{\eta_{N} \log ^{d} N} \sum_{i_{1}, \ldots, i_{d}=1}^{N-1} \int \prod_{A_{i_{1}, \cdots, i_{d}}}^{d} \frac{\sin ^{2}(N+1 / 2) x_{s}}{2 \sin \left(x_{s} / 2\right)} d x_{1} \cdots d x_{d} \\
\geq & \frac{c}{\eta_{N} \log ^{d} N} \sum_{i_{1}, \ldots, i_{d}=1}^{N-1} \frac{1}{i_{1} \cdots i_{d}} \geq \frac{c}{\eta_{N}} \rightarrow \infty
\end{aligned}
$$

as $N \rightarrow \infty$. Applying the Banach-Steinhaus Theorem, from (29) and (30) we conclude that there exists a continuous function $f \in \Lambda^{\#} B V\left(T^{d}\right)$ such that

$$
\sup _{N}\left|S_{N, \cdots, N} f(0, \cdots, 0)\right|=\infty .
$$

Theorem 4 is proved.

The next theorem follows from Theorems 3 and 4. 
Theorem 5. For any $d>1$ the class of functions $f(x), x \in T^{d}$ satisfying the following condition

$$
\sum_{n=1}^{\infty} \frac{v_{s}^{\#}(f, n) \log ^{d-1}(n+1)}{n^{2}}<\infty, \quad s=1, \ldots, d,
$$

is a class of convergence.

\section{REFERENCES}

[1] Bakhvalov, A. N. Continuity in $\Lambda$-variation of functions of several variables and the convergence of multiple Fourier series (Russian). Mat. Sb. 193, 12(2002), 3-20; English transl. in Sb. Math. 193, 11-12(2002), no. 11-12, 1731-1748.

[2] Chanturia, Z. A. The modulus of variation of a function and its application in the theory of Fourier series, Soviet. Math. Dokl. 15 (1974), 67-71.

[3] Dyachenko M. I. Waterman classes and spherical partial sums of double Fourier series, Anal. Math. 21(1995), 3-21

[4] Dyachenko M. I. Two-dimensional Waterman classes and $u$-convergence of Fourier series (Russian).Mat. Sb. 190 (1999), no.7, 23-40; English transl. in Sb. Math. 190 (1999), no.7-8, 955-972.

[5] Dyachenko M. I, Waterman D. Convergence of double Fourier series and W-classes, Trans. Amer. Math. Soc. 357 (2005), 397-407.

[6] Goginava U. On the uniform convergence of multiple trigonometric Fourier series. East J. Approx. 3 (1999), no.5, 253-266.

[7] Goginava U. Uniform convergence of Cesáro means of negative order of double WalshFourier series. J. Approx. Theory. 124 (2003), 96-108.

[8] Goginava U, Sahakian A. On the convergence of double Fourier series of functions of bounded partial generalized variation. East J. Approx. 16 (2010), no.2, 109-121.

[9] Goginava U, Sahakian A.On the convergence of multiple Fourier series of functions of bounded partial generalized variation, Anal. Math. (to appear).

[10] Hardy G. H. On double Fourier series and especially which represent the double zeta function with real and incommensurable parameters. Quart. J. Math. Oxford Ser. 37 (1906), 53-79.

[11] Jordan C. Sur la series de Fourier. C.R. Acad. Sci. Paris. 92(1881), 228-230.

[12] Marcinkiewicz J. On a class of functions and their Fourier series. Compt. Rend. Soc. Sci. Warsowie, 26 (1934), 71-77.

[13] Sablin A. I. $\Lambda$-variation and Fourier series (Russian), Izv. Vyssh. Uchebn. Zaved. Mat. 10 (1987), 66-68; English transl. in Soviet Math. (Izv. VUZ) 31 (1987).

[14] Sahakian A. A. On the convergence of double Fourier series of functions of bounded harmonic variation (Russian). Izv. Akad. Nauk Armyan. SSR Ser. Mat. 21 (1986), no.6, 517-529; English transl. in, Soviet J. Contemp. Math. Anal. 21 (1986), no.6, $1-13$.

[15] Waterman D. On convergence of Fourier series of functions of generalized bounded variation. Studia Math., 44 (1972), no.1, 107-117.

[16] Waterman D. On the summability of Fourier series of functions of $\Lambda$-bounded variation. Studia Math. 54 (1975/76), no. 1, 87-95.

[17] Wiener N. The quadratic variation of a function and its Fourier coefficients. Massachusetts J. Math., 3 (1924), 72-94.

[18] Zygmund A. Trigonometric series. Cambridge University Press, Cambridge, 1959. 
U. Goginava, Department of Mathematics, Faculty of Exact and Natural Sciences, Iv. Javakhishvili Tbilisi State University, Chavchavadze str. 1, TbilISI 0128, GeORGIA

E-mail address: zazagoginava@gmail.com

A. Sahakian, Yerevan State University, Faculty of Mathematics and MeChanics, Alex Manoukian str. 1, Yerevan 0025, Armenia

E-mail address: sart@ysu.am 